\title{
PERFORMANCE DOS FUNDOS DE INVESTIMENTO EM COTAS NO BRASIL
}

\author{
PERFORMANCE OF FUNDS OF FUNDS IN BRAZIL
}

\author{
DERMEVAL MARTINS BORGES JUNIOR \\ Mestre em Administração na Faculdade de Gestão e Negócios da \\ Universidade Federal de Uberlândia (FAGEN/UFU). Endereço: Av. \\ João Naves de Ávila, 2121 / Bloco 1F / Sala 1F-216 / Campus Santa \\ Mônica / Uberlândia/MG / Brasil. \\ E-mail: dermevaljr14@hotmail.com
}

\section{RODRIGO FERNANDES MALAQUIAS}

Doutor em Administração de Empresas pela Fundação Getúlio Vargas (EAESP/FGV). Professor do Programa de Pós-Graduação em Administração (Mestrado) da FAGEN/UFU e do Programa de PósGraduação em Ciências Contábeis da FACIC/UFU. Endereço: Av. João Naves de Ávila, 2121 | Bloco 1F / Sala 1F-216 / Campus Santa Mônica / Uberlândia/MG / Brasil.

E-mail: rodrigofmalaquias@yahoo.com.br

\section{RESUMO}

Dentre as modalidades de fundos existentes, estão os Fundos de Investimento em Cotas (FICs). São fundos que adquirem participações de outros fundos em vez de investir diretamente em ativos do mercado. Além de oferecer as vantagens inerentes aos fundos tradicionais, o FIC possibilita a expansão das opções de investimento, uma vez que sua estrutura permite acessar diversos outros fundos com baixo investimento inicial. Por outro lado, a estrutura dos FICs apresenta taxas e custos que podem afetar negativamente sua performance. Diante disso, o objetivo deste estudo consiste em analisar a performance dos FICs brasileiros, em comparação aos demais fundos. A partir de uma amostra composta por 1.723 fundos de ações, com dados disponíveis para o período de janeiro de 2005 até março de 2016, utilizou-se a análise de regressão para atingir o objetivo do trabalho. Os resultados indicaram melhores indicadores de performance (Índice de Sharpe) para os FICs, em comparação aos demais fundos da amostra.

Palavras-Chave: Investidores institucionais. Fundos de cotas. Fundos de fundos de ações. Fundos de investimento. Desempenho.

\begin{abstract}
Among the modalities of existing funds are the Investment Funds in Quotas (FICs), which they are funds that acquire shares from other funds instead of directly investing in market assets. In addition to offering the inherent advantages of traditional funds, the FIC allows the expansion of investment options, since its structure allows access to several other funds with low initial investment. On the other hand, the structure of the FICs presents rates and costs that can negatively affect their performance. However, the objective of this study is to analyze the performance of the Brazilian FICs, with focus on the other funds. From a sample of 1,723 stock funds, with data available for the period from January 2005 to March 2016, the analysis was used to reach the objective of the study. The results indicated better performance indicators
\end{abstract}


(Sharpe Ratio) for FICs, compared to the other funds in the sample.

Keywords: Institutional investors. Funds of funds. Funds of equity funds. Investment funds. Performance.

\section{INTRODUÇÃO}

A indústria de fundos no Brasil tem apresentado crescimento tanto em termos absolutos como quando comparada a outras regiões como a América Latina e os Estados Unidos, apesar de a indústria de fundos norte-americana ser, aproximadamente, 14 vezes maior que a brasileira. Esse crescimento possibilitou desenvolver e regulamentar diversas modalidades de fundos de investimento. Dentre as modalidades de fundos existentes, estão os Fundos de Investimento em Cotas (FIC), que, em vez de investirem diretamente em ativos do mercado de ações, adquirem participações de outros fundos (Varga \& Wengert, 2011).

Além de oferecerem as vantagens inerentes aos fundos tradicionais, os FICs possibilitam a expansão das opções de investimento, uma vez que sua estrutura permite que os investidores acessem diversos outros fundos, inclusive fechados, e com baixo investimento inicial. Apesar disso, apresentam custos e taxas adicionais em comparação com os fundos tradicionais diretos, pois além das taxas provenientes dos fundos subjacentes, o investidor assume ainda gastos extras referentes à gestão do FIC (Bertin \& Prather, 2009; Ang, RhodesKropf, \& Zhao, 2008).

Alguns estudos sugerem que os custos maiores dos FICs são compensados por conta da diversificação e modelo de gestão. O trabalho de Dai e Shawky (2010) evidencia que quanto mais diversificado for o FIC melhor o resultado e menor o risco. Turner (2004) destaca que o gestor desse tipo de fundo consegue adicionar valor, por meio da habilidade de escolher o melhor mix de mercados ou classe de ativos para investir e identificar os gestores de fundos individuais que estejam mais bem preparados para atingir os objetivos propostos. Apesar disso, Amenc e Vaissié (2006) observaram que apenas 20\% dos FICs analisados apresentaram criação de valor, levando em conta a construção de portfólio e gestão.

No Brasil, são poucos os trabalhos que abordam os FICs. Malaquias e Eid (2014) constataram que, em tempos de crise, os FICs conseguem agregar mais valor que outros fundos multimercados. Em consonância, Malaquias e Mamede (2015) sugeriram melhores indicadores de desempenho dos FICs, mesmo com a estrutura que envolve maiores taxas, quando comparados aos demais fundos de investimento. No que se refere ao benefício proporcionado pelos gestores de fundos, Cotrim (2012) afirma que os gestores de FICs brasileiros conseguem gerar valor ao selecionarem as estratégias que comporão a carteira do fundo, porém destroem valor ao tentarem antecipar os movimentos de mercado e ao escolherem os melhores fundos dentro de cada estratégia.

Geralmente, os FICs são incluídos nos estudos acadêmicos brasileiros como uma variável de controle (Assali, 2008; Malaquias \& Eid, 2014; Malaquias \& Mamede, 2015). Observando-se os argumentos anteriormente apresentados, os FICs apresentam uma estrutura diferente dos demais fundos de investimento, o que motivou a realização de um estudo com foco nesse tipo de investidor institucional. Dessa forma, o objetivo geral desta pesquisa consiste em analisar os fatores determinantes do retorno ajustado ao risco dos fundos de investimento em ações brasileiros, com foco nos fundos de cotas.

Conforme dados do Investment Company Institute (2014), em 2013, os ativos da indústria de fundos mútuos da América Latina somaram aproximadamente US\$1,2 trilhões, valor quase $25 \%$ superior ao observado em 2009, que foi de US\$ 905 bilhões. Vale ressaltar que a participação do Brasil nos ativos da indústria de fundos mútuos da América Latina foi de $85 \%$, em 2013, o equivalente a quase US\$ 1,02 trilhões. Destaca-se ainda que houve um crescimento expressivo desses valores, uma vez que em 2009 o Brasil contava com US\$ 784 bilhões de ativos nesses fundos.

Ainda, de acordo com os dados do Investment Company Institute (2014), ao analisar os ativos da indústria de fundos mútuos dos BRICS (Brasil, Rússia, Índia, China e África do Sul), em 2013 o valor verificado foi de US\$1,75 trilhões, quase $26 \%$ maior que em 2009 , apurado em US $\$ 1,30$ trilhões. Quanto à participação do Brasil em relação aos BRICS, em 2013, foi de 
$58,2 \%$ e em 2009 de 60,4\%. Apesar da participação do Brasil, no que se refere aos valores dos ativos dos fundos, ser muito inferior nos BRICS em comparação à América Latina, ainda assim é bastante significativa, e por isso representa um mercado relevante para ser estudado.

Vale ressaltar que os FICs estão inseridos num segmento importante do mercado financeiro brasileiro: os investidores institucionais. Essa classe de investidores é responsável por movimentar quantidades significativas de recursos no mercado financeiro, caracterizandose como os maiores investidores em fundos de investimento no Brasil (Instituto de Pesquisa Econômica Aplicada, 2015).

\section{REVISÃO DE LITERATURA}

Os fundos de investimento estão entre as principais formas de captação e alocação de recursos financeiros existentes. O volume de recursos aplicados nesse tipo de investimento cresceu expressivamente no Brasil no início da década de 1990, com o processo de liberalização da economia. Proporcionou assim, a criação de vários novos fundos e o aumento do investimento nos fundos já existentes (Milani, Ceretta, Barba, \& Casarin, 2010).

Conforme Bertin e Prather (2009), a indústria de fundos de investimento tem presenciado um rápido crescimento em uma categoria de fundos denominada fundos de investimento em cotas (FIC). Basicamente, os FICs são fundos que investem em participações de fundos tradicionais em vez de investirem diretamente no mercado financeiro. Ang et al. (2008) indicam três razões principais para o aumento da popularidade desses fundos: i) possibilidade dos investidores acessarem fundos de investimento que estão fechados para investidores individuais; ii) investimento mínimo necessário menor quando comparado com outros fundos; iii) acesso a portfólio diversificado, de modo que apenas investidores individuais com grande quantidade de capital conseguiriam replicar tal nível de diversificação.

Segundo Fothergill e Coke (2001), os FICs oferecem ao investidor uma ampla gama de alternativas de estratégias de investimento em função da combinação de diferentes fundos subjacentes na carteira do FIC, de modo que tal diversificação pode proporcionar retornos com baixos níveis de riscos. Além disso, por conta da estrutura dos FICs, investidores individuais tendem a obter maiores benefícios em termos de transparência do que alcançariam caso investissem em um fundo de investimento de modo direto, minimizando assim eventuais problemas com a escolha do fundo ou com o processo de due diligence, por exemplo.

Apesar desses benefícios, é importante destacar que os FICs apresentam custos e taxas adicionais em comparação aos fundos tradicionais, o que os torna mais caros. Conforme Ang et al. (2008), os FICs repassam aos investidores todas as taxas cobradas pelos fundos subjacentes na carteira do fundo de cotas. Além disso, existem taxas extras para compensar o gestor do fundo, as quais também são relevantes e podem corroer uma eventual performance acima do retorno esperado.

Ammann e Moerth (2008), ao compararem o desempenho de fundos de hedge tradicionais e FICs de fundos de hedge, observaram que no período de janeiro de 1994 a abril de 2005 o retorno médio anual dos fundos de hedge foi de $8,42 \%$, enquanto que os FICs apresentaram 6,53\% de retorno médio anual para o mesmo período. Os autores atribuíram essa diferença de performance justamente à estrutura de taxas dos FICs, de modo que os resultados sugeriram uma incidência de taxas adicionais de 1,84\% por ano em comparação aos fundos de hedge diretos, número muito próximo da diferença entre o desempenho médio anual ( $1,89 \%$ ao ano).

Quanto à rentabilidade, vale destacar alguns estudos sobre FICs de private equity, como o de Aggarwal, Sharma e Prashar (2012), que, ao relacionar três índices de FICs de private equity com cinco índices de mercado (AORD, SSE Composite, N225, FTSE 100 e S\&P500), evidenciou que dois dos três índices de FICs demonstraram maiores retornos anuais do que quatro dos índices de mercado em quatro dos cinco anos analisados. Gresch e Wyss (2011), ao analisarem 1.641 fundos de private equity, indicaram que os FICs são mais atrativos no que se refere à relação risco e retorno do que os fundos de investimento diretos.

Por outro lado, Denvir e Hutson (2006), ao analisarem dados de 332 FICs, no período de janeiro de 1990 a maio de 2003, verificaram pior desempenho em comparação a fundos de hedge individuais, por conta das taxas elevadas inerentes à estrutura dos FICs. Apesar disso, 
os autores também evidenciaram características valiosas dos FICs que compensam esse problema, como, por exemplo, o fato de seus retornos não sofrerem da mesma assimetria negativa que caracteriza o retorno de fundos de hedge.

No caso dos FICs brasileiros, os resultados do estudo de Malaquias e Mamede (2015) sugeriram melhores indicadores de desempenho em comparação aos demais fundos de investimento, mesmo com a estrutura que envolve maiores taxas.

Similarmente, Rocco (2009) estudou a rentabilidade, volatilidade, patrimônio líquido e o retorno ajustado ao risco de fundos multimercados brasileiros, comparando os resultados de fundos diretos e FICs no período de 2002 a 2007. Dentre os resultados, Rocco (2009) encontrou evidências de maior Índice de Sharpe para fundos de investimento em cotas de outros fundos em relação a fundos que investem diretamente em ativos do mercado.

Adicionalmente, Malaquias e Eid (2014), ao analisarem fundos multimercados brasileiros, verificaram que os FICs, mesmo com a estrutura com maiores incidências de taxas do que nos fundos tradicionais, conseguem agregar mais valor do que os demais fundos multimercados analisados. A justificativa indicada para isso é que os FICs conseguem encontrar oportunidades de negociação previstas em um segmento que não se mostra de acordo com a Hipótese de Eficiência de Mercado. Vale ressaltar ainda que, conforme os autores, o tamanho dos fundos, independentemente do período analisado, apresentou relação positiva com a performance, ou seja, quanto maior o fundo melhor o seu desempenho.

De acordo com Geranio e Zanotti (2005), os FICs possuem a maior taxa de administração em comparação com as outras categorias de fundos. Essa taxa reflete os custos com a forma pela qual a gestão dos fundos é feita. Os FICs que investem em sistemas pela sua mesma empresa gestora de ativos cobram apenas uma comissão, enquanto os que compram fundos em outra empresa gestora adicionam taxa de administração extra para compensar os serviços de alocação de ativos. Os autores ainda indicaram duas explicações para as taxas mais altas dos FICs: i) um preço premium por conta do benefício proporcionado pela diversificação; ii) a predominância de fundos de cotas que investem em sistemas não geridos pelo mesmo promotor. Esses argumentos levam a pensar que a relação da performance com a taxa de administração dos FICs pode apresentar um comportamento diferente daquele apresentado nos fundos tradicionais.

Alguns estudos sugerem que, embora os FICs sejam mais caros, isso pode ser compensado pelos benefícios proporcionados por conta da diversificação. Em comparação aos fundos de investimento tradicionais, a pesquisa de Bertin e Prather (2009) indicou, durante o período de 1996 a 2003, desempenho favorável, principalmente por conta da diversificação dos gestores de fundos. O estudo ainda apontou que a identificação da equipe de gestores e o conhecimento de gestão influenciam no desempenho FICs.

Ao comparar o desempenho entre fundos de cotas especializados (diversificam apenas entre gestores, mas ficam dentro de uma única estratégia de fundo) e diversificados (diversificam tanto entre gestores quanto de estratégias de fundo), Dai e Shawky (2010) encontraram evidências de melhores resultados e menor risco quanto maior a diversificação. Amo, Harasty e Hillion (2007) também estudaram os benefícios da diversificação nos FICs. Seus resultados indicaram que ao adicionar apenas um pequeno número de fundos de hedge, não mais que seis, o risco do fundo de cotas de hedge cai pela metade em comparação com fundos de hedge individuais.

Em discordância aos trabalhos anteriores sobre diversificação, conforme Brown, Gregoriou e Pascalau (2012), a redução do risco proporcionada pelos FICs tende a acabar quando atinge entre 10 a 20 fundos de hedge subjacentes. Além disso, a diversificação acima de 25 fundos subjacentes leva, na maioria dos casos, a uma redução significativa no desempenho. Desse modo, percebe-se que existem limites relevantes no que tange à diversificação proporcionada pelos FICs.

Brands e Gallagher (2005) também afirmam que, independentemente da estratégia de seleção de portfólio, a maioria dos ganhos de diversificação dos FICs são obtidos com um portfólio que compreende apenas seis fundos. Após esse ponto, os aumentos incrementais no número de fundos mantidos no portfólio não apresentam ganhos significativos do ponto de vista do risco e retorno. 
Ainda, no que se refere ao portfólio dos fundos, Milan e Eid (2014) analisaram a rotatividade das carteiras de fundos de investimento em ações brasileiros no período de 2007 a 2011, a fim de identificar eventuais efeitos no desempenho. Os principais resultados expostos pelos autores indicaram que níveis elevados de rotatividade mensal das carteiras exercem efeito negativo sobre a performance. Além disso, os FICs apresentaram índices de rotatividade mensais significativamente menores do que os fundos de ações, sugerindo, portanto, que os FICs tendem a manter suas carteiras de ativos estáveis.

Outro fator que pode representar uma importante vantagem para os FICs refere-se à escolha do gestor. Borges e Martelanc (2015), a partir de dez mil simulações com dados, provenientes de fundos de investimento de ações brasileiros no período de 2000 a 2013, encontraram evidências consistentes da existência de habilidade dos gestores de fundos em produzir retornos anormais positivos, isto é, superiores aos que seriam obtidos por pura sorte, especialmente em gestores de grandes fundos.

Nesse contexto, Reddy, Brady e Patel (2007) afirmam que os FICs conseguem escolher gestores de um grande universo de fundos, enquanto que um gestor multi-estratégia, por exemplo, fica limitado a sua habilidade de contratar equipes conforme cada estratégia que participa. Assim, de certa forma, é possível afirmar que um FIC tem acesso aos melhores gestores para uma ampla gama de estratégia de investimentos. Isso pode representar um impacto positivo no desempenho do fundo, caso consiga montar um portfólio com gestores acima da média.

Turner (2004) destaca que ao investir em um FIC, diferentemente do que ocorre em um fundo de investimento comum, o gestor do fundo de cotas consegue adicionar valor por meio da habilidade de escolher o melhor mix de mercados ou classe de ativos para investir. Identifica os gestores de fundos individuais que estão mais bem preparados para entregar valor nesses mercados, de modo a atingir os objetivos de investimento do fundo de cotas.

Nesse sentido, Aiken, Clifford e Ellis (2015) examinaram as carteiras dos fundos de fundos de hedge, a fim de identificar os canais por meio dos quais os FICs adicionam valor para seus clientes. Apesar dos mencionados autores identificarem carteiras mantidas pelos FICs compostas por diversos fundos, cujas quais seriam dispendiosas e inviáveis para investidores individuais gerenciarem por conta própria, as evidências sugeriram que os FICs não possuem habilidade extraordinária na escolha de seus fundos de hedge subjacentes. Em outras palavras, os FICs podem aplicar recursos em fundos com fraco desempenho futuro. Por outro lado, Aiken et al. (2015) demonstraram que os FICs são hábeis nas decisões de término de investimento, ou seja, depois que um FIC liquida sua posição em um fundo de hedge, posteriormente tende a apresentar pior desempenho (mais frequentemente). Assim, Aiken et al. (2015) entendem que os FICs exercem papel fundamental como intermediários nos mercados em que os custos de transação são significantes.

Em contrapartida, Cotrim (2012), ao estudar fundos de fundos de hedge brasileiros, classificados como fundos multigestor, verificou que os gestores desses fundos conseguem gerar valor ao selecionarem as estratégias que comporão a carteira do respectivo fundo multigestor, porém destroem valor ao tentarem antecipar os movimentos de mercado e, além disso, não conseguem escolher os melhores fundos dentro de cada estratégia.

Os resultados da pesquisa de Kat e Helder, apresentados em Gregoriou (2006), indicaram que a maioria dos FICs, em termos de retorno aos investidores, não demonstraram adicionar valor, o que não significa que esses fundos não tenham utilidade, uma vez que devido às vantagens descritas anteriormente, como baixo investimento necessário, permitem que pequenos investidores tenham acesso a portfólio de fundos diversificado. O estudo de Amenc e Vaissié (2006) mostrou que aproximadamente $89 \%$ dos FICs demonstraram criar valor em termos de construção de portfólio e 31\% apresentaram adicionar valor por meio de gestão ativa. Contudo, ao considerar ambas, construção de portfólio e gestão, apenas $20 \%$ apresentaram criação de valor.

Edelman, Fung, Hsieh e Naik (2012) utilizaram uma base de dados de 1.591 FICs de hedge para verificar o desempenho de portfólios de fundos de hedge diversificados durante o período de janeiro de 2005 até dezembro de 2010. Seus resultados demonstraram que os retornos dos FICs analisados são, em grande parte, impulsionados pela exposição a fatores de risco sistemático. Além disso, os FICs apresentaram retorno médio acumulado positivo, quando 
expostos a diferentes fontes de risco sistemático, apenas no primeiro período analisado (compreendido entre janeiro de 2005 a junho de 2007).

De acordo com Leusin (2006), apesar de os FICs apresentarem muitas vantagens para o investidor, como diversificação, escolha dos profissionais que irão gerir os fundos, acesso do pequeno investidor a fundos restritos, entre outros, o efeito das taxas incidentes gera um custo muito elevado para os cotistas, de modo a restringir a atratividade desses investimentos. Os resultados do trabalho de Leusin (2006) indicaram que, embora os gestores consigam agregar valor ao investidor, o excesso de taxas compromete muito o retorno, corroendo grande parte da vantagem desse tipo de investimento.

Conforme Füss, Kaiser e Strittmatter (2009), características como tamanho e idade também podem influenciar o desempenho risco/retorno dos fundos. Os resultados mostraram que o retorno diminui para pequenos FICs e aumenta para grandes FICs, quando a experiência (idade) dos fundos cresce. A explicação para isso é que FICs novos tendem a ser mais cautelosos no risco tomado, mais diversificados e ter volatilidade total de retorno menor do que os mais velhos. Shawky e Wang (2014) também verificaram que os maiores tendem a ter melhor desempenho que os fundos menores, isso porque como os FICs não geram portfólios de ativos diretos, eles estão menos sujeitos aos custos de liquidez associados a portfólios elevados de ativos em outros tipos de investimentos.

Outro aspecto que merece destaque refere-se aos períodos de crise. Existem evidências na literatura de que períodos de crise podem exercer algum efeito sobre a performance dos fundos de investimento. Em seu estudo sobre fundos multimercados brasileiros, Malaquias e Eid (2014) estabeleceram uma variável para verificar eventual influência dos períodos de crise sobre o desempenho dos fundos, de modo que o período de janeiro de 2005 a maio de 2008 foi considerado um momento de mercado em alta e o intervalo temporal entre junho de 2008 a agosto de 2011 um período de crise. Os resultados da pesquisa de Malaquias e Eid (2014), nesse contexto, demonstraram diferenças estatisticamente significativas entre a performance média dos fundos em períodos de crise e em períodos de estabilidade. Nos períodos de crise o desempenho médio dos fundos foi pior.

Similarmente, Joaquim e Moura (2011) analisaram desempenho e indicadores de persistência de fundos multimercados brasileiros no período compreendido entre 2007 e 2011 , observando os efeitos que a crise de 2008 poderia exercer na performance. Dentre os resultados, os autores verificaram, mesmo no período de crise, a existência de retornos anormais e persistência para uma quantidade significativa de fundos em nível agregado, porém poucos fundos demonstraram persistência de desempenho em nível individual, de modo que o desempenho decresceu conforme aumento no horizonte temporal.

Garay, Hernández e Rivillo (2017) analisaram o comportamento de variáveis microeconômicas de fundos multimercados durante o período anterior à crise de 2008, com o objetivo de identificar se tais elementos poderiam predizer a probabilidade de sobrevivência dos FICs no período de crise. Dentre os resultados do estudo, foi observado que a probabilidade de sobrevivência dos FICs durante a crise financeira global de 2008 pode ser explicada pelo comportamento da estimativa de retorno médio, do desvio padrão dos retornos mensais, da taxa de administração, da taxa de performance e do coeficiente de curtose dos retornos mensais.

De maneira geral, é possível perceber que parece ser consenso entre os estudos que os FICs tendem a ser menos rentáveis quando comparados com a rentabilidade dos fundos de investimento tradicionais, principalmente por conta de sua estrutura, que apresenta taxas adicionais. Apesar disso, alguns trabalhos indicam que o fato pode ser compensado por meio de outros benefícios proporcionados, principalmente pela diversificação e gestão desses fundos. Mesmo assim, não se pode afirmar que tais fatores são benefícios incontestáveis, uma vez que, como visto, a diversificação possui limites e há controvérsias quanto ao valor criado pelos gestores, o que motiva e mostra a oportunidade de se realizar um novo estudo sobre o assunto. 


\section{PROCEDIMENTOS METODOLÓGICOS}

Para a realização deste estudo, foram considerados todos os fundos de ações com dados mensais disponíveis no banco de dados Economática para o período de janeiro de 2005 até março de 2016. O ano de 2005 foi escolhido em razão de utilizar o período após a harmonização do conteúdo divulgado pelos fundos de investimento (Instrução CVM n. 409, n. 411 , n. 413, 2004). A data final foi selecionada em razão de ser o mês com dados mais recentes disponíveis durante o período de coleta (que se encerrou em julho/2016), o que proporcionou 1.723 fundos disponíveis. É importante destacar que a amostra engloba fundos em atividade, bem como fundos que foram encerrados durante o período.

Foram incluídos no estudo quatro fatores que trabalhos anteriores já apontaram ter uma relação significativa com a performance. São eles:

i) taxa de administração: considerada nos estudos de Dai e Shawky (2010), Sialm, Sun e Zheng (2013) e Malaquias e Eid (2014). Espera-se encontrar neste trabalho uma relação negativa entre a taxa de administração e a rentabilidade, uma vez que a incidência dessas taxas não está necessariamente atrelada ao bom desempenho de determinado fundo e, por isso, tendem a corroer os ganhos. Forma de mensuração: taxa máxima de administração cobrada pelos fundos anualmente;

ii) taxa de performance: considerada nos estudos de Brown, Goetzmann e Liang (2003), Dai e Shawky (2010) e Malaquias e Eid (2014). Diferentemente do que ocorre com as taxas de administração, as taxas de performance representam um incentivo aos gestores dos fundos para obter bom resultado. Assim, espera-se encontrar neste estudo uma relação positiva entre a taxa de performance e o desempenho. Forma de mensuração: variável dummy, recebendo 1 para fundos que cobram taxa de performance e zero para os demais;

iii) tamanho do fundo: considerado nos estudos de Füss et al. (2009), Shawky e Wang (2014), Malaquias e Eid (2014) e Malaquias e Mamede (2015). Espera-se encontrar neste trabalho uma relação positiva entre o tamanho do fundo e o desempenho, tendo em vista que fundos maiores tendem a possuir menores custos operacionais por conta de economias de escala. Forma de mensuração: logaritmo neperiano do Patrimônio Líquido médio do fundo durante o período de observação;

iv) idade do fundo: considerada nos estudos de Agarwal e Kale (2007), Füss et al. (2009), Li, Zhang e Zhao (2011) e Malaquias e Mamede (2015). Com base nesses estudos, existem evidências tanto para uma relação positiva entre idade e performance, tendo em vista que os fundos novos apresentam custos iniciais com a construção do portfólio e maior cautela nos investimentos, como também para uma relação negativa, uma vez que podem apresentar melhor desempenho por estarem mais propensos a utilizar estratégias de investimento inovadoras, com o intuito de se estabelecerem no mercado. Forma de mensuração: idade, em anos, com base na última observação disponível para cada fundo.

A Tabela 1 apresenta uma síntese das variáveis em estudo:

Tabela 1

\begin{tabular}{l|l|l}
\begin{tabular}{l} 
Variáveis em estudo \\
\multicolumn{1}{c|}{ Variável }
\end{tabular} & \multicolumn{1}{c}{ Descrição } & \multicolumn{1}{c}{ Literatura } \\
\hline ind_sharpe & Índice de Sharpe & $\begin{array}{l}\text { Joaquim e Moura (2011), Milan e } \\
\text { Eid (2014) e Malaquias e Eid (2014). }\end{array}$ \\
\hline fic & $\begin{array}{l}\text { dummy para fundo de cotas (fic), recebendo 1 para } \\
\text { fundos de cotas e 0 para os demais }\end{array}$ & $\begin{array}{l}\text { Assali (2008), Malaquias e Eid } \\
(2014) \text { e Malaquias e Mamede } \\
(2015)\end{array}$ \\
\hline taxa_adm & $\begin{array}{l}\text { taxa máxima de administração cobrada pelos fundos } \\
\text { anualmente }\end{array}$ & $\begin{array}{l}\text { Dai e Shawky (2010), Sialm, Sun e } \\
\text { Zheng (2013) e Malaquias e Eid } \\
\text { (2014). }\end{array}$ \\
\hline taxa_perf & $\begin{array}{l}\text { variável dummy, recebendo 1 para fundos que cobram } \\
\text { taxa de performance zero para os demais }\end{array}$ & $\begin{array}{l}\text { Brown, Goetzmann e Liang (2003), } \\
\text { Dai e Shawky (2010) e Malaquias e } \\
\text { Eid (2014). }\end{array}$ \\
\hline tamanho & $\begin{array}{l}\text { logaritmo neperiano do Patrimônio Líquido médio do do } \\
\text { fundo durante o período de observação }\end{array}$ & $\begin{array}{l}\text { Füss et al. (2009), Shawky e Wang } \\
\text { (2014), Malaquias e Eid (2014) e } \\
\text { Malaquias e Mamede (2015) }\end{array}$ \\
\hline idade & $\begin{array}{l}\text { idade, em anos, com base na última observação } \\
\text { disponível para cada fundo }\end{array}$ & $\begin{array}{l}\text { Agarwal e Kale (2007), Füss et al. } \\
\text { (2009), Li et al. (2011) e Malaquias e } \\
\text { Mamede (2015) }\end{array}$ \\
\hline
\end{tabular}

Fonte: Elaborado pelos autores. 
Para ajustar a performance pelo risco, foi utilizado o Índice de Sharpe, que indica o retorno médio acima da taxa livre de risco, ponderado pela volatilidade dos retornos. A estimativa para taxa livre de riscos utilizada neste estudo foi a rentabilidade mensal da SELIC. Assim, a variável dependente do modelo quantitativo é o output do Índice de Sharpe, para cada fundo. A principal variável independente é a dummy para fundo de cotas (fic), recebendo 1 para fundos de cotas e 0 para os demais. As outras variáveis independentes são aquelas já apresentadas: tamanho, idade, taxa de administração e taxa de performance. $O$ modelo quantitativo elaborado está indicado a seguir:

$$
\text { ind_sharpe } e_{i}=\beta_{0}+\beta_{1} \text { fic }_{i}+\beta_{2} \text { tamanho }_{i}+\beta_{3} \text { taxa_adm }{ }_{i}+\beta_{4} \text { taxa_perf }_{i}+\beta_{5} \text { idade }_{i}+\varepsilon_{i}
$$

em que, ind_sharpe representa o Índice de Sharpe no período; fic uma variável dummy para fundo de cotas, recebendo 1 para fundos de cotas e 0 para os demais; tamanho representa 0 logaritmo neperiano do Patrimônio Líquido médio dos fundos; taxa_adm representa a taxa máxima de administração cobrada pelos fundos; taxa_perf representa uma variável dummy, recebendo 1 para fundos que cobram taxa de performance e zero para os demais; idade representa a idade dos fundos em anos.

É oportuno indicar que foi realizada uma análise adicional, incluindo a segregação do modelo em dois diferentes períodos econômicos. Para a escolha dos períodos, inicialmente foi calculado o prêmio pelo risco mensal de todos os fundos da amostra. Em seguida, calculou-se, por ano, o prêmio médio pelo risco. Observou-se que os anos de 2008, 2011, 2013, 2014 e 2015 apresentaram prêmio médio pelo risco negativo, enquanto que nos demais períodos o prêmio médio pelo risco foi positivo. Dessa forma, as informações dos referidos períodos $(2008,2011,2013,2014$ e 2015) foram consideradas como dentro de períodos de crise.

O modelo quantitativo foi estimado com base na análise de regressão multivariada com erros padrão robustos a heterocedasticidade (uma vez que o teste de White foi estatisticamente significativo nos modelos estimados neste estudo - Tabelas 3, 4 e 5). Segundo Hair, Black, Babin, Anderson e Tatham (2009), a análise de regressão multivariada consiste em uma técnica estatística usada para examinar a relação entre uma única variável dependente e um conjunto de variáveis independentes, com ampla aplicabilidade em problemas de pesquisa que visam à previsão e à explicação.

\section{RESULTADOS}

A Tabela 2, a seguir, apresenta a estatística descritiva para as variáveis do estudo. 0 Índice de Sharpe médio aponta que o valor adicionado pelos fundos, em média, não supera a taxa livre de risco de cada período (pois sua média é igual a -0,093). Aproximadamente $40 \%$ da amostra é composta por fundos de cotas $(39,6 \%)$ e $47 \%$ deles cobram taxa de performance de seus cotistas.

Tabela 2

\section{Estatística descritiva}

\begin{tabular}{|c|c|c|c|c|c|}
\hline Variável & $\mathbf{n}$ & Média & Desv. Pad. & Mín. & Máx. \\
\hline tamanho & 1.723 & 16,933 & 1,503 & 12,204 & 22,299 \\
\hline fic & 1.723 & 0,396 & a & 0,000 & 1,000 \\
\hline taxa_adm & 1.723 & 1,601 & 1,237 & 0,000 & 10,000 \\
\hline taxa_perf & 1.723 & 0,470 & $a$ & 0,000 & 1,000 \\
\hline idade & 1.723 & 6,916 & 5,913 & 1,017 & 49,456 \\
\hline ind_sharpe & 1.723 & $-0,093$ & 0,156 & $-0,945$ & 0,801 \\
\hline
\end{tabular}

A Tabela 3 contém os resultados para a análise de regressão, com o objetivo de se testar a hipótese do estudo. Essa análise envolve todos os fundos da amostra (ou seja, não possui o viés de sobrevivência, por não desconsiderar fundos fechados no período). Os 
resultados indicaram que todas as variáveis utilizadas foram importantes para entender as variações do Índice de Sharpe dos fundos, pois foram estatisticamente significativas até o nível de $5 \%$. Os resultados também indicaram a ausência de problemas decorrentes de multicolinearidade, uma vez que o teste VIF ficou abaixo de 5.

Tabela 3

Resultados para a análise de regressão (todos os fundos)

\begin{tabular}{lcccc}
\hline Variável & beta & $\begin{array}{c}\text { erro padrão } \\
\text { (robusto) }\end{array}$ & $\mathbf{t}$ & sig. \\
\hline tamanho & 0.033 & 0.003 & 12.320 & 0.000 \\
taxa_adm & -0.012 & 0.003 & -4.020 & 0.000 \\
taxa_perf & -0.017 & 0.007 & -2.420 & 0.016 \\
idade & 0.004 & 0.001 & 6.470 & 0.000 \\
fic & 0.034 & 0.007 & 4.640 & 0.000 \\
constante & -0.658 & 0.045 & -14.500 & 0.000 \\
\hline
\end{tabular}

Nota. R-quadrado ajustado $=17,02 \%$. Máximo valor para a estatística VIF: 1,29. Os valores extremos para a variável dependente (nesse caso, $5 \%$ do banco de dados) foram excluídos e o modelo foi estimado novamente; os coeficientes, em termos de sinal e nível de significância, foram equivalentes. Indicaram que os resultados não parecem estar sendo influenciados por potenciais efeitos de valores extremos.

Fonte: Elaborado pelos autores.

De maneira geral, os resultados indicaram melhores indicadores de performance para os Fundos de Investimento em Cotas (FICs) em comparação aos demais fundos da amostra. Tais evidências estão em discordância com alguns trabalhos observados na literatura sobre o tema, como os de Denvir e Hutson (2006), Gregoriou (2006), Leusin (2006), Ang et al. (2008) e Edelman et al. (2012), os quais demonstraram pior desempenho dos FICs. Porém, é importante ressaltar que os resultados verificados nesta pesquisa estão em conformidade com os estudos recentes desses fundos no mercado brasileiro, como os apresentados por Malaquias e Eid (2014) e Malaquias e Mamede (2015). Bertin e Prather (2009) também constataram melhor desempenho dos FICs em relação aos fundos tradicionais.

Conforme já comentado, todas as variáveis de controle apresentaram relação significativa com a variável dependente. Assim como os resultados dos trabalhos de Füss et al. (2009), Malaquias e Eid (2014), Shawky e Wang (2014) e Malaquias e Mamede (2015), foi verificada neste estudo uma relação positiva entre o tamanho do fundo, medido por seu patrimônio líquido, e a performance, isto é, o retorno ajustado ao risco é menor para fundos pequenos e maior para fundos grandes. Os fundos maiores tendem a conseguir economias de escala que podem impactar em melhor desempenho. Além disso, a melhor performance dos fundos grandes pode ser também um reflexo dos benefícios proporcionados pela maior diversificação, conforme os estudos de Amo et al. (2007), Bertin e Prather (2009) e Dai e Shawky (2010).

Ao considerar a idade dos fundos, os resultados foram convergentes aos encontrados por Füss et al. (2009) e Malaquias e Mamede (2015), uma vez que foi encontrada uma relação positiva entre a idade e o retorno, de modo que os fundos novos não apresentaram melhor desempenho do que os fundos antigos. O raciocínio de que os fundos novos estão mais propensos a buscar alternativas de investimento arrojadas na tentativa de obter melhores resultados do que os fundos já estabelecidos, para que assim consigam ganhar espaço no mercado, não parece ser aderente às características dos fundos da amostra deste estudo. A análise quantitativa também apresentou indícios de que as taxas de administração e taxas de performance estão relacionadas com menor performance alcançada pelos fundos.

Como o objetivo de analisar se os resultados seriam equivalentes considerando-se apenas os fundos em atividade, os fundos fechados durante o período foram excluídos da amostra e o modelo foi estimado novamente (total de fundos em atividade: 1.370). A Tabela 4 contém os resultados e indica que o modelo com todos os fundos apresenta melhor poder de explicação (o r-quadrado ajustado do primeiro modelo é superior). 
Tabela 4

Resultados para a análise de regressão (apenas fundos sobreviventes)

\begin{tabular}{lcccc}
\hline Variável & Beta & $\begin{array}{c}\text { erro padrão } \\
\text { (robusto) }\end{array}$ & t & sig. \\
\hline tamanho & 0.027 & 0.003 & 10.470 & 0.000 \\
taxa_adm & -0.012 & 0.003 & -3.920 & 0.000 \\
taxa_perf & -0.012 & 0.007 & -1.710 & 0.088 \\
idade & 0.003 & 0.001 & 5.380 & 0.000 \\
fic & 0.027 & 0.007 & 3.680 & 0.000 \\
constante & -0.560 & 0.045 & -12.490 & 0.000
\end{tabular}

Nota. R-quadrado ajustado $=13,87 \%$. Máximo valor para a estatística VIF: 1,31. Os valores extremos para a variável dependente (nesse caso, $5 \%$ do banco de dados) foram excluídos e o modelo foi estimado novamente; os coeficientes, em termos de sinal e nível de significância, foram equivalentes, indicando que os resultados não parecem estar sendo influenciados por potenciais efeitos de valores extremos.

Fonte: Elaborado pelos autores.

Os resultados apresentados na Tabela 4 são equivalentes àqueles apresentados na Tabela 4, exceto pela variável Taxa de Performance, que deixou de ser significativa ao nível de $5 \%$, mas continua significativa ao nível de 10\%. A análise da principal hipótese do estudo continua a mesma: fundos de cotas registraram melhor indicador de retorno ajustado ao risco. Tendo em vista os resultados apresentados, percebe-se que, mesmo possuindo uma estrutura com maior incidência de taxas, os FICs conseguem se destacar no seu desempenho perante os outros tipos de fundos. Assim sendo, podem representar uma alternativa oportuna para os investidores, pois além da sua performance superior, observada neste trabalho, possuem benefícios incomuns em demais tipos de investimento, como acesso a fundos fechados, baixo investimento inicial, acesso a portfólio diversificado, entre outros. Com o objetivo de verificar potenciais efeitos de períodos de crise nos resultados deste estudo, foi realizada uma nova análise de regressão, cujos resultados estão disponíveis na Tabela 5.

Tabela 5

Resultados para a análise de regressão (apenas fundos sobreviventes, considerando o controle por diferentes períodos)

\begin{tabular}{lcccc} 
Variável & beta & $\begin{array}{c}\text { erro padrão } \\
\text { (robusto) }\end{array}$ & t & sig. \\
\hline crise & -0.615 & 0.022 & -27.700 & 0.000 \\
tamanho & 0.024 & 0.008 & 2.920 & 0.004 \\
taxa_adm & -0.021 & 0.009 & -2.270 & 0.023 \\
taxa_perf & 0.030 & 0.022 & 1.320 & 0.186 \\
idade & -0.005 & 0.002 & -3.090 & 0.002 \\
fic & 0.138 & 0.027 & 5.130 & 0.000 \\
constante & -0.066 & 0.135 & -0.490 & 0.624 \\
\hline
\end{tabular}

Nota. R-quadrado ajustado $=24,23 \%$. Máximo valor para a estatística VIF: 1,31 . Crise $=$ variável dummy que recebe 1 para os Índices de Sharpe no período de 2008, 2011, 2013, 2014 e 2015, recebendo 0 para os Índices de Sharpe obtidos nos demais períodos. Os valores extremos para a variável dependente (nesse caso, $5 \%$ do banco de dados) foram excluídos e o modelo foi estimado novamente; os coeficientes, em termos de sinal e nível de significância, foram equivalentes, indicando que os resultados não parecem estar sendo influenciados por potenciais efeitos de valores extremos.

Fonte: Elaborado pelos autores.

Os resultados disponíveis na Tabela 5 estão coerentes com a literatura (Malaquias \& Eid, 2014), de que os períodos de crise podem afetar de maneira significativa a performance dos fundos. Entretanto, mesmo com o controle por períodos de crise, as variáveis Tamanho e Fundos de Cotas continuaram apresentando-se com efeito positivo e estatisticamente significativo.

Por outro lado, ressalva-se que, tanto na Tabela 3 como na Tabela 4, os coeficientes encontrados, apesar de estatisticamente significativos, podem não apresentar significância econômica. Diante disso, foram estimados o D de Cohen para as variáveis analisadas 
considerando a diferença entre grupos de fundos de investimento tradicionais (não-FICs) e FICs, conforme indicado na Tabela 6.

Tabela 6

Tamanho de efeito por grupo (não-FICs e FICs)

\begin{tabular}{lccc}
\hline Variável & D de Cohen & \multicolumn{2}{c}{ Intervalo de Confiança (95\%) } \\
\hline ind_sharpe & $-0,172$ & $-0,269$ & $-0,754$ \\
Tamanho & $-0,016$ & $-0,113$ & 0,080 \\
taxa_adm & $-0,535$ & $-0,633$ & $-0,437$ \\
taxa_perf & $-0,143$ & $-0,239$ & $-0,046$ \\
idade & $-0,054$ & $-0,151$ & 0,042 \\
\hline
\end{tabular}

Nota. observações de não-FICs = 1.040; observações de FICs = 683 .

Fonte: Elaborado pelos autores.

De acordo com os resultados apresentados na Tabela 6, observa-se que, em média, os FICs demonstraram Índice de Sharpe superior em comparação aos outros fundos da amostra, de modo que o tamanho do efeito estimado pelo $D$ de Cohen pode ser classificado como mediano $(-0,172)$. Isso quer dizer que, em termos práticos, há alguma significância econômica de desempenho superior dos FICs, suprimindo a possibilidade de eventuais resultados enganosos indicados na análise de regressão, provenientes do tamanho elevado da amostra.

Deve-se mencionar que, ainda no que se refere à análise do tamanho de efeito, os FICs apresentaram, em média, taxas mais altas do que os demais fundos. O tamanho do efeito estimado pelo $D$ de Cohen foi classificado como grande para a taxa de administração $(-0,535)$ e pequeno para a taxa de performance $(-0,143)$. Esses resultados corroboram trabalhos anteriores que já demonstraram empiricamente a incidência de taxas superiores nos FICs em função de sua estrutura, tais como Geranio e Zanotti (2005), Denvir e Hutson (2006), Ang et al. (2008), Ammann e Moerth (2008). O tamanho de efeito observado para as variáveis idade ($0,054)$ e tamanho $(-0,016)$ parece não ter sido economicamente significativo.

\section{CONCLUSÃO}

Este trabalho foi desenvolvido com o objetivo geral de analisar a performance dos fundos de investimentos em cotas, considerando um cenário com argumentos favoráveis e contrários à geração de valor extraordinário desses fundos, especialmente em decorrência de uma estrutura que pode envolver dupla cobrança de taxas. A base de dados englobou 1.723 fundos de ações e os resultados encontrados foram testados na amostra completa, bem como em uma subamostra, apenas com os fundos sobreviventes no período. Os resultados nessas duas amostras foram equivalentes para o principal teste de hipótese do estudo. Observando-se estudos anteriores, quatro variáveis de controle foram também adicionadas ao estudo, quais sejam: tamanho e idade, que apresentaram relação positiva com o retorno ajustado ao risco, e taxas de administração e de performance, que apresentaram relação negativa com a performance.

O principal resultado indicou que os FICs pertencentes à amostra do estudo apresentaram melhores índices de retorno ajustado ao risco, o que está alinhado com argumentos já disponíveis na literatura, como nos estudos de Turner (2004), Dai e Shawky (2010) e Malaquias e Mamede (2015). As evidências obtidas neste estudo também sugerem a possível vantagem dos FICs no que diz respeito à gestão dos fundos. Muitas críticas são feitas por conta do seu elevado custo de gestão, uma vez que, além das taxas de administração dos fundos subjacentes, podem existir taxas extras para compensar o gestor do fundo de cotas. $O$ fato de esta pesquisa indicar melhor retorno ajustado ao risco dos FICs, em comparação aos fundos tradicionais, aponta evidências de que as estratégias de gestão por parte dos gestores de fundos de cotas podem favorecer a identificação de ativos com potencial de superar o benchmark, estratégias essas que podem funcionar bem no segmento de fundos de investimento (em que os fundos de cotas atuam). 
Ao avaliar se essa diferença de desempenho entre os FICs e os demais fundos da amostra mostrou-se expressiva em termos econômicos, os resultados dos testes de tamanho de efeito indicaram que, no que diz respeito à diferença entre as médias do Índice de Sharpe, o tamanho do efeito encontrado foi classificado como mediano, ou seja, corroboraram os achados da análise de regressão. Do mesmo modo, os testes de tamanho de efeito indicaram que os FICs cobram taxas maiores em relação aos fundos tradicionais, especialmente taxas de administração.

Este trabalho avança em relação aos demais que já foram desenvolvidos sobre o assunto, sobretudo, ao propor uma análise que tem como foco especificamente os FICs, os quais são normalmente preteridos das amostras dos estudos de fundos de investimentos no Brasil. Além disso, contribui para a literatura da área ao indicar resultados que podem respaldar discussões futuras a respeito do potencial desses fundos como alternativa de investimento, inclusive quando comparados aos fundos tradicionais.

A principal limitação relacionada a este estudo encontra-se na base de dados, que considera apenas a classe de fundos de ações, quando existem outros tipos de fundos de gestão ativa no Brasil, como é o caso dos fundos multimercados. Nos fundos multimercados, há também a possibilidade de operação dos FICs, e novos estudos sobre o assunto podem ser desenvolvidos para verificar se os resultados são aderentes ou não aos encontrados nesta pesquisa.

\section{REFERÊNCIAS}

Agarwal, V. \& Kale, J. R. (2007). On the relative performance of multi-strategy and funds of hedge funds. Journal of Investment Management, 5(3), 41.

Aggarwal, R., Sharma, M., \& Prashar, E. (2012). Fund of Funds Company Indices: Performance Analysis and Maximizing the Coefficient of Determination. The Journal of Private Equity, 16(1), 100.

Aiken, A. L., Clifford, C. P., \& Ellis, J. (2015). The Value of Funds of Hedge Funds: Evidence from Their Holdings. Management Science, 61(10), 2415-2429.

Amenc, N. \& Vaissié, M. (2006). Determinants of Funds of Hedge Funds' Performance. The Journal of Investing, 15(4), 46-52.

Ammann, M. \& Moerth, P. (2008). Performance of Funds of Hedge Funds. The Journal of Wealth Management, 11(1), 46-63.

Amo, A. V., Harasty, H., \& Hillion, P. (2007). Diversification benefits of funds of hedge funds: Identifying the optimal number of hedge funds. The Journal of Alternative Investments, 10(2), 10.

Ang, A., Rhodes-Kropf, M. \& Zhao, R. (2008). Do funds-of-funds deserve their fees-onfees? (No. w13944). National Bureau of Economic Research.

Assali, N. A. (2008). Análise de desempenho e características de fundos de fundos multigestores do mercado Brasileiro no período de setembro/1998 a Agosto/2007. Dissertação de Mestrado em Finanças e Economia Empresarial, Escola de Economia de São Paulo da Fundação Getúlio Vargas - EESP-FGV, São Paulo, SP, Brasil.

Bertin, W. J. \& Prather, L. (2009). Management structure and the performance of funds of mutual funds. Journal of business research, 62(12), 1364-1369.

Borges, E. C. \& Martelanc, R. (2015). Sorte ou habilidade: uma avaliação dos fundos de investimento no Brasil. Revista de Administração, 50(2), 196-207.

Brands, S. \& Gallagher, D. R. (2005). Portfolio selection, diversification and fund-of-funds: a note. Accounting \& Finance, 45(2), 185-197.

Brown, S. J., Goetzmann, W. N., \& Liang, B. (2003). Fees on fees in funds of funds (No. w9464). National Bureau of Economic Research. 
Brown, S. J., Gregoriou, G. N., \& Pascalau, R. (2012). Diversification in funds of hedge funds: Is it possible to overdiversify?. Review of Asset Pricing Studies, 2(1), 89-110.

Cotrim, F. M. (2012). Um estudo sobre a capacidade de gestores de fundos multigestor adicionarem valor aos cotistas. Dissertação de Mestrado em Finanças e Economia Empresarial, Escola de Pós-Graduação em Economia da Fundação Getúlio Vargas EPGE-FGV, Rio de Janeiro, RJ, Brasil.

Dai, N. \& Shawky, H. A. (2010). Diversification Strategies and the Performance of Funds of Hedge Funds. Available at SSRN 1719749.

Denvir, E. \& Hutson, E. (2006). The performance and diversification benefits of funds of hedge funds. Journal of International Financial Markets, Institutions and Money, 16(1), 4-22.

Edelman, D., Fung, W., Hsieh, D. A., \& Naik, N. Y. (2012). Funds of hedge funds: performance, risk and capital formation 2005 to 2010. Financial Markets and Portfolio Management, 26(1), 87-108.

Fothergill, M. \& Coke, C. (2001). Funds of Hedge Funds: An Introduction to Multi-Manager Funds. The Journal of Alternative Investments, 4(2), 7-16.

Füss, R., Kaiser, D. G., \& Strittmatter, A. (2009). Measuring funds of hedge funds performance using quantile regressions: Do experience and size matter?. The Journal of Alternative Investments, 12(2), 41.

Garay, U., Hernández, M., \& Rivillo, C. (2017). Variables microeconómicas de los fondos de fondos de cobertura (FFC) y su desempeño durante la crisis financiera global 2008-2009. Revista Finanzas y Política Económica, 9(2), 373-396.

Geranio, M. \& Zanotti, G. (2005). Can mutual funds characteristics explain fees?. Journal of Multinational Financial Management, 15(4), 354-376.

Gregoriou, G. N. (2006). Funds of Hedge Funds: Performance, Assessment, Diversification, and Statistical Properties. Elsevier, 2011.

Gresch, N. \& Von Wyss, R. (2011). Private Equity Funds of Funds vs. Funds: A Performance Comparison. The Journal of Private Equity, 14(2), 43.

Hair, J. F., Jr., Black, W. C., Babin, B. J., Anderson, R. E., \& Tatham, R. L. (2009). Análise multivariada de dados. Porto Alegre: Bookman.

Instituto de Pesquisa Econômica Aplicada. (2015). Os fundos de investimento no Brasil de 2008 a 2013: institucionalidades e interfaces com a política econômica.

Instrução CVM n. 409, de 18 de agosto de 2004. Dispõe sobre a constituição, a administração, o funcionamento e a divulgação de informações dos fundos de investimento. Rio de Janeiro: Comissão de Valores Mobiliários.

Instrução CVM n. 411, de 26 de novembro de 2004. Altera a Instrução CVM n. 409, de 18 de agosto de 2004. Rio de Janeiro: Comissão de Valores Mobiliários.

Instrução CVM n. 413, de 30 de dezembro de 2004. Altera a Instrução CVM n. 409, de 18 de agosto de 2004. Rio de Janeiro: Comissão de Valores Mobiliários.

Investment Company Institute (US). (2014). Investment Company Fact Book. Investment Company Institute. Recuperado de https://www.ici.org/pdf/2014_factbook.pdf

Joaquim, G. P. G. \& Moura, M. L. (2011). Performance and Persistence of Brazilian Hedge Funds during the Financial Crisis. Revista Brasileira de Finanças, 9(4), 465-488.

Leusin, L. M. C. (2006). Análise de Fundo de Fundos: Um estudo para o caso brasileiro (Dissertação de Mestrado Profissional em Finanças e Economia Empresarial). Escola de Pós-Graduação em Economia da Fundação Getúlio Vargas - EPGE-FGV, Rio de Janeiro, RJ, Brasil. 
Li, H., Zhang, X., \& Zhao, R. (2011). Investing in talents: Manager characteristics and hedge fund performances. Journal of Financial and Quantitative Analysis, 46(01), 59-82.

Malaquias, R. F. \& Eid, W., Jr. E. (2014). Fundos multimercados: desempenho, determinantes do desempenho e efeito moderador. Revista de Administração Mackenzie, 15(4), 135.

Malaquias, R. F. \& Mamede, S. D. P. N. (2015). Efeito Calendário e Finanças Comportamentais no Segmento de Fundos Multimercados. Revista de Administração Contemporânea, 19, 98.

Milan, P. L. A. B. \& Eid, W., Jr. (2014). Elevada Rotatividade de Carteiras e o Desempenho dos Fundos de Investimento em Ações. Revista Brasileira de Finanças, 12(4), 469-497.

Milani, B., Ceretta, P. S., Barba, F. G. de., \& Casarin, F. (2010). Fundos de Investimento Brasileiros: a influência dos momentos superiores na avaliação de desempenho. Revista Brasileira de Gestão e Negócios, 10(36), 289-303.

Reddy, G., Brady, P., \& Patel, K. (2007). Are Funds of Funds Simply Multi-Strategy Managers with Extra Fees?. The Journal of Alternative Investments, 10(3), 49.

Rocco, L. U. (2009). Uma análise de desempenho dos fundos multimercados com renda variável e alavancagem no Brasil durante o período entre 2002-2007. Monografia de Conclusão de Curso de Ciências Econômicas, Universidade Federal de Santa Catarina UFSC, Florianópolis, SC, Brasil.

Shawky, H. A. \& Wang, Y. (2014). Liquidity Risk and Economies of Scale in Funds of Hedge Funds. The Journal of Alternative Investments, 17(2), 51.

Sialm, C., Sun, Z., \& Zheng, L. (2013). Home bias and local contagion: Evidence from funds of hedge funds (No. w19570). National Bureau of Economic Research.

Turner, C. (2004). International Funds: A practical guide to their establishment and operations. Elsevier, Oxford.

Varga, G. \& Wengert, M. (2011). A indústria de fundos de investimentos no Brasil. Revista de Economia e Administração, 10(1). 